\title{
Perturbative Propagation Models for Coherent Systems
}

\author{
P. Serena and A. Bononi \\ Dip. Ing. informazione, Università degli Studi di Parma, Parma, Italy \\ paolo.serena@unipr.it
}

\begin{abstract}
We review the basics of perturbation models and discuss the main implications in modeling the variance of the nonlinear interference. A modulation format dependent theoretical model for the perturbative interference is proposed.

OCIS codes: (060.2330) Fiber optics communications; (060.1660) Coherent communications.
\end{abstract}

\section{Introduction}

Perturbation theory, i.e., the study of the impact of small parameters on mathematical models, found wide application in the analysis of the nonlinear Schrödinger equation (NLSE). If the perturbative solution is a Taylor series in the small parameter, we refer to a regular perturbation (RP) solution of the problem [1-6]. It can be shown that the same solution can be obtained by using alternative techniques, like the Volterra series expansion [4], the first order Picard iteration, and the exponential Euler integrator method.

Perturbative models of the NLSE recently captured the attention of the community for two main aspects: i) their closed form expressions stimulate applications in digital equalization of nonlinear impairments, e.g., [7]; ii) the statistical moments of the nonlinear interference (NLI) can be successfully evaluated $[2,3,5,8]$.

Aim of this paper is to review the basics of RP and to shed light on the application in statistical modeling, by providing an equivalent but alternative expression for the modulation-format-dependent power spectral density (PSD) given by the enhanced Gaussian noise (EGN) model [8]. A comparison with the GN model [2] is given.

\section{RP, eRP, LP}

The NLSE for propagation of a field $A$ is expressed in terms of operators as:

$$
\frac{\partial A}{\partial z}=(\mathscr{L}-j \gamma \mathscr{N}) A
$$

with $\mathscr{L}=-\frac{\alpha}{2}+j \frac{\beta_{2}}{2} \frac{\partial^{2}}{\partial t^{2}}$ and $\mathscr{N}(A)=|A|^{2} A$. Assuming $z$-independent parameters for the sake of simplicity, using the integrating factor $A=e^{\mathscr{L} z} U$ we can rewrite (1) in integral form as [1,5]:

$$
U(z)=U(0)-j \gamma \int_{0}^{z} e^{-\mathscr{L} \xi} \mathscr{N}\left(e^{\mathscr{L} \xi} U\right) \mathrm{d} \xi \triangleq U_{0}+U_{1}
$$

Such an equation can be solved iteratively by using $U_{k+1}$ and $U_{k}$ in place of $U(z)$ in the left/right hand side, respectively. Since $\mathscr{N}$ is normally small, RP is stopped at the first iteration at the first order (RP1). A block diagram sketching this idea is shown in Fig. 1(left), and compared with the standard discretization of the split step Fourier method (SSFM). While each block $L$ accounts for $e^{\mathscr{L} z}$, in each path only one nonlinear block $\mathscr{N}$ is crossed, according to the RP1 idea. The RP method can be applied as well in wavelength division multiplexing (WDM) and polarization division multiplexing (PDM); in particular, the cross channel interference of channel $\vec{B}=e^{j \bar{\omega} t}\left[B_{x}, B_{y}\right]^{T}$ on $\vec{A}=\left[A_{x}, A_{y}\right]^{T}$ can be accounted for by substituting $\mathscr{N}$ with $\mathscr{N}_{A B}=\frac{8}{9}\left(\|\vec{A}\|^{2}+\vec{B} \vec{B}^{\dagger}+\vec{B} \dagger \vec{B}\right) \vec{A}$, where $\dagger$ indicates transpose conjugate. Assuming a linearly modulated digital signal with initial supporting pulse $p(0, t)$, by defining $p(z, t)=e^{\mathscr{L} z} p(0, t)$, and matched filter impulse response $p^{*}(z,-t)$, the block diagram of the RP method takes the form of Fig. 1 (right) [3,5].

$\mathrm{RP}$ forces the nonlinear perturbation to be additive. This is generally not true, and some advanced methods were introduced to cope with it. A first basic attempt is eRP, which exploits RP in a reference system that tracks the nonlinear average phase rotation $\phi(z)$, thus removing the average of the secular terms in the perturbation. This corresponds to using $A=e^{\mathscr{L} z} e^{j \phi(z)} U$, where $\phi(z)$ can be estimated by $\phi(z)=\operatorname{Im}\left[\left\langle U_{1} / U_{0}\right\rangle\right],\langle$.$\rangle indicating averaging. The second$ advanced method forces the perturbation to be logarithmic, i.e., $U(z)=U_{0} \exp \left(U_{1} / U_{0}\right)[9,10]$. Both eRP and LP are a posteriori manipulations of the RP solution, of which they share the same complexity. We verified by simulation that they both over-estimate the Q-factor [11], and that LP is never more accurate than eRP in practical WDM systems, both dispersion-unmanaged (DU) and dispersion-managed (DM). Recently, Fan et al. proposed a mixed RP-LP approximation [12]. 

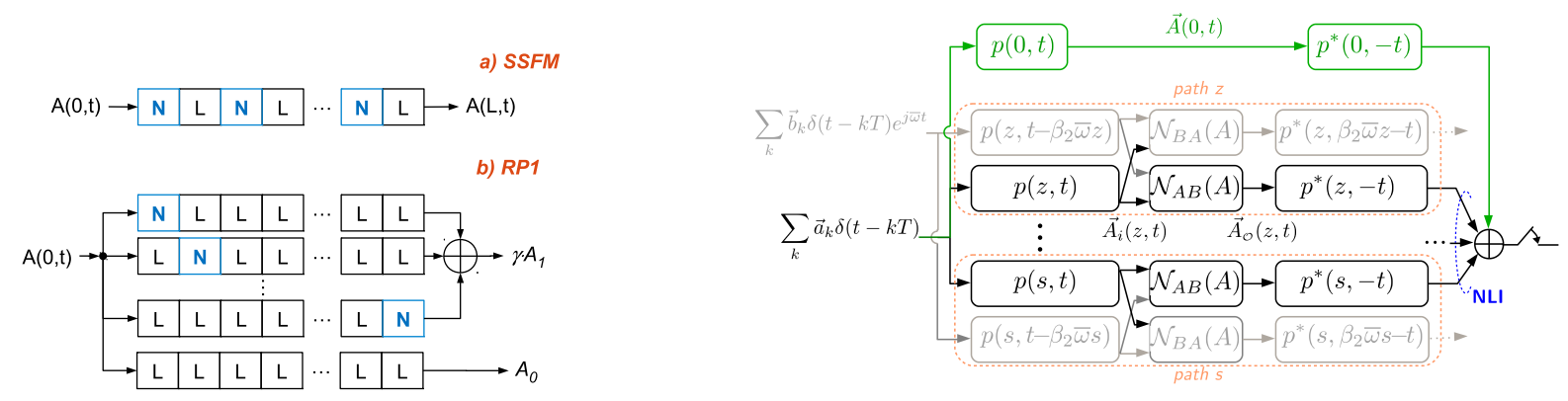

Fig. 1. Block diagram of the RP method. Left: comparison with SSFM. Right: Example of application in WDM with matched filtering. $p(z, t)$ : digital signal supporting pulse at time $t$ linearly impaired after $z \mathrm{~km}$.

\section{Application to Statistical Modeling}

The RP1 algorithm of Fig. 1 does not provide significant computational savings compared to SSFM. Indeed, it can be exploited to evaluate the correlation function of the NLI. The block diagram of Fig. 1 (right) suggests the way. For the sake of simplicity, we set the idea in single-polarization and single channel (extensions to WDM and PDM are straightforward). The NLI is an infinite sum of contributions, thus, to get the NLI auto-correlation we need the crosscorrelation between any NLI outgoing paths $z$ and $s$. Along such paths, relating the input/output correlation across any linear block is just a matter of linear system theory [13]. The challenge regards nonlinear blocks. i.e., relating $R_{z s}^{i}(t, \tau)$ to $R_{z s}^{\mathscr{O}}(t, \tau)$, with $R_{z s}^{i, \mathscr{O}}(t, \tau) \triangleq E\left[A_{i, \mathscr{O}}(z, t+\tau) A_{i, \mathscr{O}}^{*}(s, t)\right]$ (see Fig. 1(right)). Under the key GN-model assumption of a stationary Gaussian input [2], in the alternative GN model derivation [13] it was shown that such a relation takes the remarkably simple form:

$$
R_{z s}^{\mathscr{O}}(\tau)=2 \mathscr{N}\left(R_{z s}^{i}(\tau)\right) .
$$

Mecozzi et al. showed instead that one can consider the actual digital signal $A(0, t)$, and derive the true NLI statistics [5]. With a digital input we deal with cyclo-stationary signals, which become almost stationary with supporting Nyquist pulses. This way, since the nonlinearity is cubic, $R_{z s}^{\sigma}(\tau)$ becomes a function of sixth order moments, which were fully evaluated by Carena et al. by working in the frequency domain [8]. The resulting model was called EGN. By operating instead in the time domain, as in [13], we get the EGN-alternative but equivalent result:

$$
R_{z s}^{\mathscr{O}}(\tau)=\frac{\mu_{2}^{3}}{T^{3}} 2|Q(z, s, \tau)|^{2} Q(z, s, \tau)+\frac{\kappa_{2 ; 2}}{T^{2}}\left\{4 F_{4}(z, s, \tau)+Q_{4}(z, s, \tau)\right\} Q(z, s, \tau)+\frac{\kappa_{3 ; 3}}{T} Q_{6}(z, s, \tau)
$$

with $T$ the symbol time, $\kappa_{2 ; 2}=\mu_{4} \mu_{2}-2 \mu_{2}^{3}$ and $\kappa_{3 ; 3}=\mu_{6}+12 \mu_{2}^{3}-9 \mu_{4} \mu_{2}$ symbol cumulants, $\mu_{n} \triangleq E\left[\left|a_{k}\right|^{n}\right]$, and:

$$
\begin{aligned}
Q(z, s, \tau) \triangleq p(z, \tau) \otimes p^{*}(s,-\tau), & Q_{4}(z, s, \tau) \triangleq p^{2}(z, \tau) \otimes\left(p^{*}(s,-\tau)\right)^{2} \\
F_{4}(z, s, \tau) \triangleq|p(z, \tau)|^{2} \otimes|p(s,-\tau)|^{2}, & Q_{6}(z, s, \tau) \triangleq|p(z, \tau)|^{2} p(z, \tau) \otimes\left\{|p(s,-\tau)|^{2} p^{*}(s,-\tau)\right\} .
\end{aligned}
$$

$\otimes$ indicates convolution. Note that with $R_{z s}^{i}(\tau)=\frac{\mu_{2}}{T} Q(z, s, \tau)$ in (3) we get the 1st term in (4), thus called GN-term. The NLI correlation function is finally related to (4) by a double integral after [13, eq. (8)]. Eq. (4) provides a physical insight about the behavior along $z$. In particular, we find that while the GN-term is dominant, the modulation format dependent terms are dominated by $F_{4}$. At $z=s, Q$ is invariant along distance, contrary to the other terms, which indeed fade out for increasing $z$. This proves that for $z \gg 1$ only the GN-part survives, thus explaining the findings in [2].

\section{Numerical Checks}

For a $5 \times 100 \mathrm{~km}$ spans, DU link with $D=17 \mathrm{ps} / \mathrm{nm} / \mathrm{km}$, and five 32 Gbaud PDM channels (spacing $37.5 \mathrm{GHz}$ ), with either QPSK or Gaussian symbols, Fig. 2 shows the NLI PSD evaluated by using Monte Carlo simulations and theory based on (4). The excellent match is an indication of the correctness of (4), and also confirms the PSD over-estimation by the GN model [8]. The theoretical NLI eRP variance can be used to analytically evaluate the system reach. We did it in [14] for a 15-channel link, and report it here in Fig. 2 (right). As discussed in [14], despite the non-negligible error of the GN model in predicting the PSD, hence the variance, the error in reach is limited. In any case, the EGN model provides a much better agreement with SSFM results (symbols). 

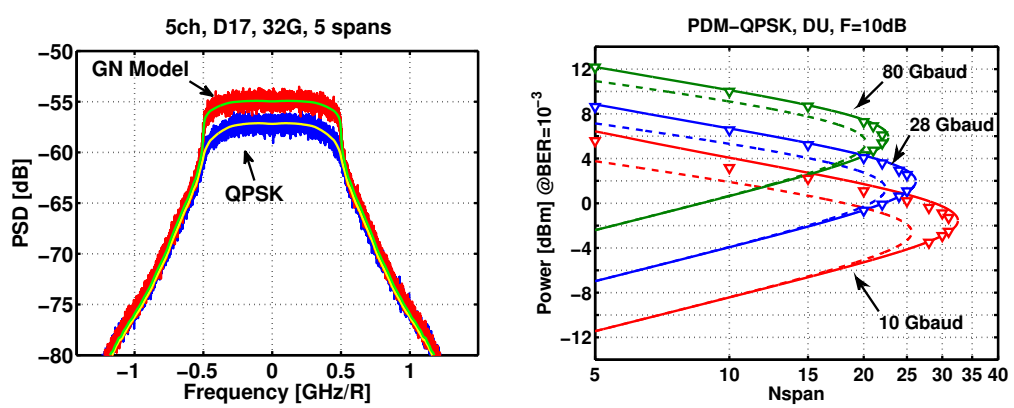

Fig. 2. Left: NLI PSD by Monte Carlo simulations or by theory (green lines). Right: Power vs. Number of span for a 15-channel PDM-QPSK system. Symbols: SSFM ; Solid: EGN ; Dashed: GN . Amplifiers noise figure: $10 \mathrm{~dB}$.
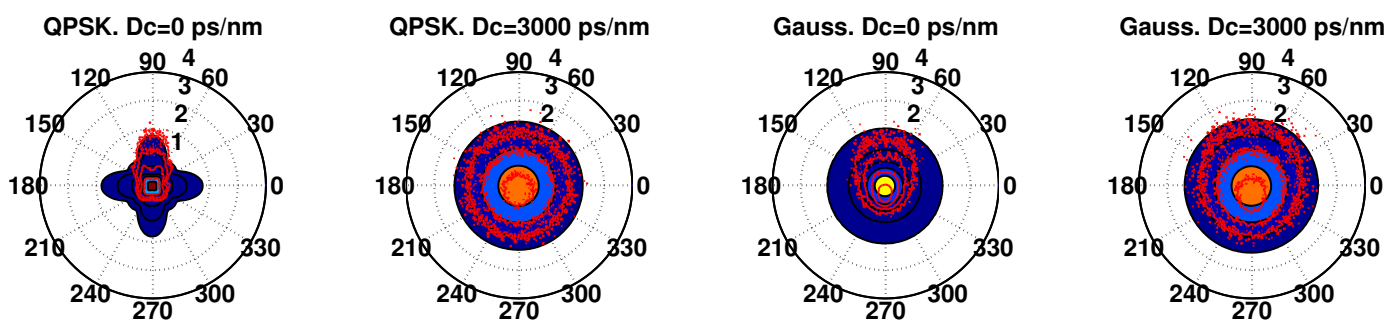

Fig. 3. Blue: eRP1 PDF Contour levels of the NLI along path $z$ of Fig. 1 cumulating a dispersion $D_{c}$. Red: contour levels of the eRP1 term at time $n=0$ conditioned to symbol $a_{n}=1$.

The EGN model looses accuracy in short, weakly dispersive links [8]. To investigate the reason, we simulated the NLI probability density function (PDF) at the output of one branch (e.g., path $z$ ) in Fig. 1(right). Results are reported in Fig. 3 for single channel. Blue contours refer to the PDF estimated using either random QPSK symbols for $a_{k}$, or Gaussian distributed $a_{k}$. The last case exactly matches the Gaussian assumption of the GN model, and not surprisingly the PDF is circularly distributed whatever the cumulated dispersion $D_{\mathrm{c}}$. However, both the GN and the EGN take averages over all input symbols, neglecting that the NLI should actually be conditioned on the symbol of interest. In fact the error probability is $P_{e}=\sum_{k} P_{r}\left(\operatorname{error} \mid a_{k}\right) P_{r}\left(a_{k}\right)$, hence what we really need is the NLI PDF conditioned on the symbol of interest. Red contours in Fig. 3 report the NLI PDF conditioned on $a_{n}=1$. It is worth noting that even by assuming Gaussian statistics for $a_{k}, k \neq n$, the conditioned PDF is not circular at small $D_{\mathrm{c}}$. This aspect is missed by both the GN and EGN models, where the forced stationarity for the NLI corresponds to averaging over all possible symbol sequences. At large $D_{\mathrm{c}}$ all symbols indeed experience the same conditional NLI PDF, thus the EGN model can be successfully applied to get the system performance.

\section{References}

1. A. Mecozzi et al. "Analysis of intrachannel nonlinear effects in highly dispersed optical pulse transmission," PTL, 12(4), p.392, (2000).

2. P. Poggiolini et al., "The GN-Model of Fiber Non-Linear Propagation and its Applications", JLT 32(4), p.3857, (2012).

3. R. Dar et al., "Properties of nonlinear noise in long, dispersion-uncompensated fiber links", Opt. Exp. 21(22), p.25685, (2013).

4. A. Vannucci et al., "The RP method: a new tool for the iterative solution of the nonlinear Schroedinger equation," JLT, 20(7), 1102, (2002).

5. A. Mecozzi et al., "Nonlinear Shannon limit in pseudolinear coherent systems", JLT 30(12), p.2011, (2012).

6. J. Tang, "A comparison study of the Shannon channel capacity of various nonlinear optical fibers," JLT 24(5), p. 2070, (2006).

7. Y. Gao et al., "Reducing the complexity of perturbation based nonlinearity pre-compensation using symmetric EDC and pulse shaping," Opt. Exp. 22(2), p.1209, (2014).

8. A. Carena et al. ,'EGN model of non-linear fiber propagation," Opt. Expr. 22(13), p.16335, (2014)

9. B. Xu et al., "Modified Volterra series transfer function method," PTL, 14(1), p.47, (2002).

10. E. Ciaramella et al., "Analytical approximation of nonlinear distortions," PTL 17(1), p.91, (2005).

11. P. Serena et al., "On the Accuracy of the Gaussian Nonlinear Model for Dispersion-unmanaged Coherent Links," ECOC 13, Th.1.D.3.

12. Y. Fan et al., "Modulation Format Dependent Phase Noise Caused by Intra-channel Nonlinearity," ECOC 12, We.2.C.3.

13. P. Serena et al., "An alternative approach to the Gaussian noise model and its system implications", JLT 31(22), p.3489, (2013).

14. P. Serena et al., "The Impact of the Modulation Dependent Nonlinear Interference Missed by the Gaussian Noise Model," ECOC 14 , Mo.4.3.1. 